\title{
Denoising of MRI Brain Images using Adaptive Clahe Filtering Method
}

\author{
P. G. Akila, K. Batri, G. Sasi, R. Ambika
}

\begin{abstract}
Image processing is a method of making the quality of an image better after removing unwanted information from image in various applications and domains to process computer effectively. Enhancement is, used to improve the quality effects of an image for further analysis. Enhancement of image can be done by filtering, de noising and contrast enhancement. Even though contrast enhancement of images is applied in different fields it is used effectively in the medical field. Medical Imaging is now recently used in most of the applications like Radiography, MRI, Nuclear medicine, Ultrasound Imaging, Tomography, Cardiograph, and Fundus Imagery and so on. The main problem in analysis of medical images is the poor contras in medical image analysis the detection of tumor, cancerous cells, malignant or benign has to be classified effectively. In this paper various spatial domain techniques and their effectiveness in terms of quality improvement are discussed. The measuring metrics used for comparing different methods are parameters Peak Signal to Noise Ratio (PSNR) and Mean Square Error (MSE), DICE coefficient, etc,.
\end{abstract}

Keywords-filter, PSNR, dice co efficient, transformation

\section{INTRODUCTION}

Contrast enhancement plays an important role in medical image enhancement to perform adjustment on darkness or lightness of the image. It mainly used to bring out the unexpressed feature in an image or increase the contrast of low contrast image for medical analysis by contrast enhancement technique. Contrast enhancement can be done in both spatial and frequency domain techniques where medical image enhancement is showing better excellence in spatial domain techniques. Very initial technique in spatial domain is Histogram equalization (HE) which is simple and efficient. Spatial domain method, a method works on the pixels of the input images. Other spatial domain techniques includes gray level transformations such as logarithmic transforms, power law transforms, linear contrast stretching and histogram equalization (HE) and point operation/space filter enhancement methods (smoothing and sharpening filters )are based on the direct manipulation of the pixels in the image [2] and the techniques like image smoothing(linear filters like neighborhood medium filter, Gaussian filter averaging filter, median filter to remove salt and pepper noise, adaptive median, wiener filter and non linear filter such as max value filter, least value filter ) and sharpening (Laplacian operator, Sobel operator, canny,

Revised Manuscript Received on October 18, 2019.

Mrs. P.G.Akila, Assistant Professor, PSNA College of Engineering and Technology, Anna University Chennai.

K. Batri, Assistant Professor, PSNA College of Engineering and Technology, Anna University Chennai.

G. Sasi, Assistant Professor, PSNA College of Engineering and Technology, Anna University Chennai.

R. Ambika, Assistant Professor, PSNA College of Engineering and Technology, Anna University Chennai.
Prewitt operator )are to emphasize the edge by neighborhood pixel manipulation. Spatial techniques involve in the change of gray level values in each pixel individually and therefore the improves contrast of the whole image. $\mathrm{HE}$ is the most efficient technique in contrast enhancement [13] in high speed with implementation simple [2]. In the field of medicine, Digital Imaging has rapid application and with the implementation of suitable algorithms for processing of Images in detection, Screening and classification of diseases. Medical images are affected by noise, blurriness and may suffer due to poor contrast and low sharpness .It may lead to false diagnosis. The detailed study of these spatial domain techniques for medical images is done here with suitable brain images.

A. Data Set

The real CT image database obtained from the Brain web Includes MRI data of more than 10 patients with each dataset containing more than 600 slices per set.

\section{II.SPATIAL DOMIAN METHODS}

\section{A .Power law transformation}

Logarithmic transformation is a contrast enhancement method that improves the pixel value (contrast) of the darker regions of the images more compared to the brighter regions to get the details in the lower intensity regions. The $\log$ transformation can be implemented as given

$$
\begin{aligned}
& s=c^{*} \log (1+\text { double }(r) \\
& s=c^{*} r^{\gamma}
\end{aligned}
$$

where $s$ is the output image, $r$ is the intensity of an input image, and $c$ is a constant and the value depends upon the limit of the grey scale window used. By varying the value of the constant $c$, the brightness of the image can be changed. The higher the value of $c$ the image will appear brighter. Thus, the $\log$ function produces too bright values to be displayed. The shape of the logarithmic curve only depends upon the range of values on which it is applied.

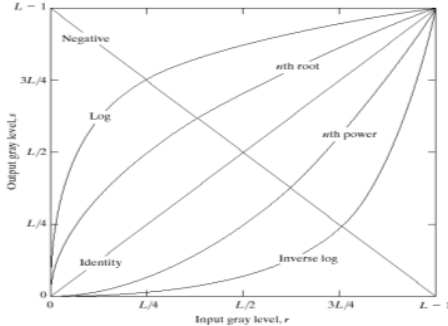

Fig 1: Power law transformation graph This is achieved by expanding the dark pixel values and compressing the bright pixel values. The only problem of the logarithmic transformation is that it over enhances the intensity of the image.

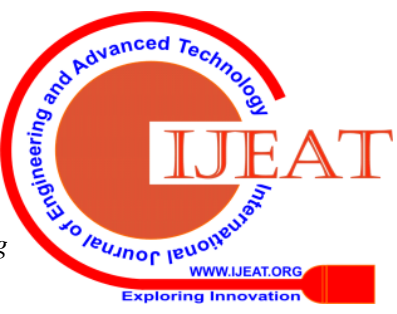




\section{Denoising of MRI Brain Images Using Adaptive Clahe Filtering Method}

The algorithm is summarized as:

Step1: Let $I(i, j)$ be the intensity values of the input CT image.

Step2: Double the image using im2double (I)

Step3: Apply the log transformation on the density values of the input image.
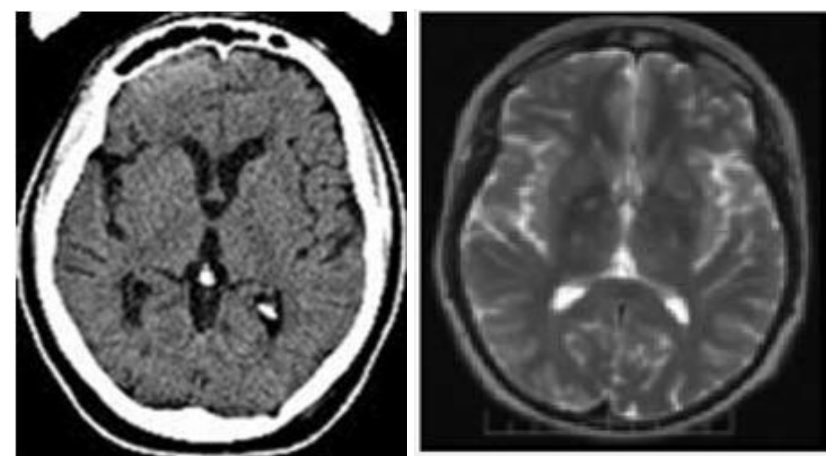

Fig 2 a) Input image b) Power law transformation output image

\section{B.Histogram Equalization}

Histogram is a diagrammatic representation of the intensity distribution of an image. In simple terms, it shows the number of pixels for each intensity value considered.
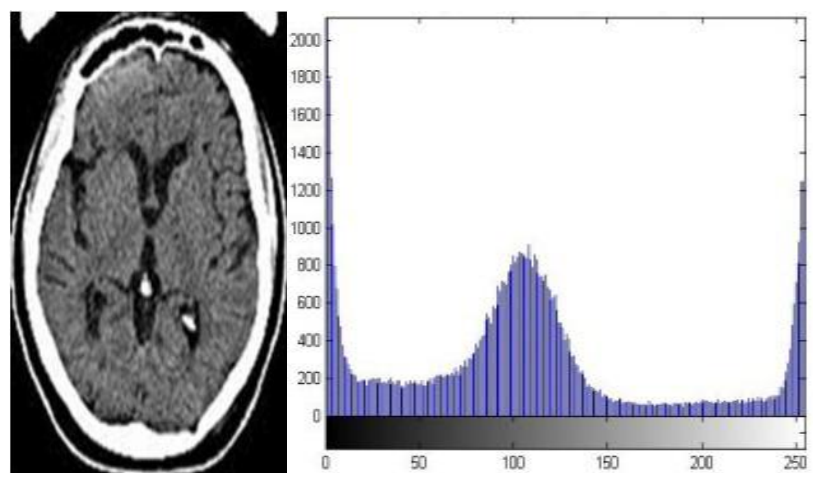

Fig 3a) Histogram of output image

Histogram Equalization is a computer image processing technique used to improve contrast in images by effectively radiating out the most common intensity values, i.e. ranging out the intensity range of the image. This method makes lower local contrast to gain a higher contrast. Many researchers have done modifications in this $\mathrm{HE}$ to reduce the drawbacks of HE. The evaluation of HE are DSIHE, MMBEBHE, RSWHE, DHE, BPDHE, CLAHE, CEDHE, BBHE.etc.
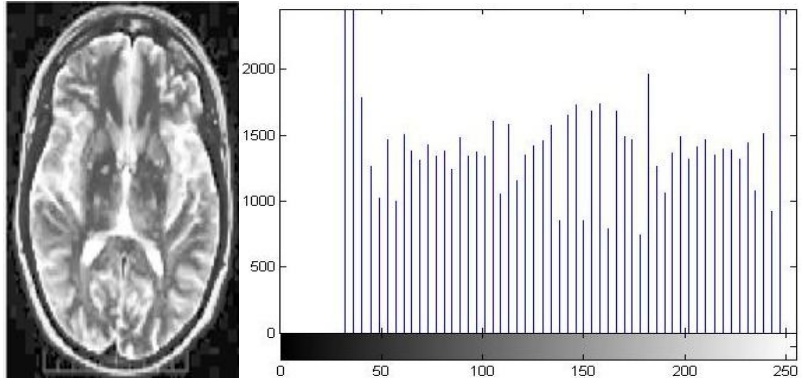

Fig 4: Histogram equalization of output image

\section{C.Contrast stetching}

Contrast stretching is a common technique in medical imaging, by ranging the pixel values according to the transformation and results in such higher density areas appearing darker.

Step1: Determine the gray level.

Step2: Compute new gray levels by $l=\left(l_{\max }-l_{\min }\right)\left(m-m_{\min }\right)+l_{\min } /\left(m_{\max }-m_{\min }\right)$

Step 3: Repeat the step 2.
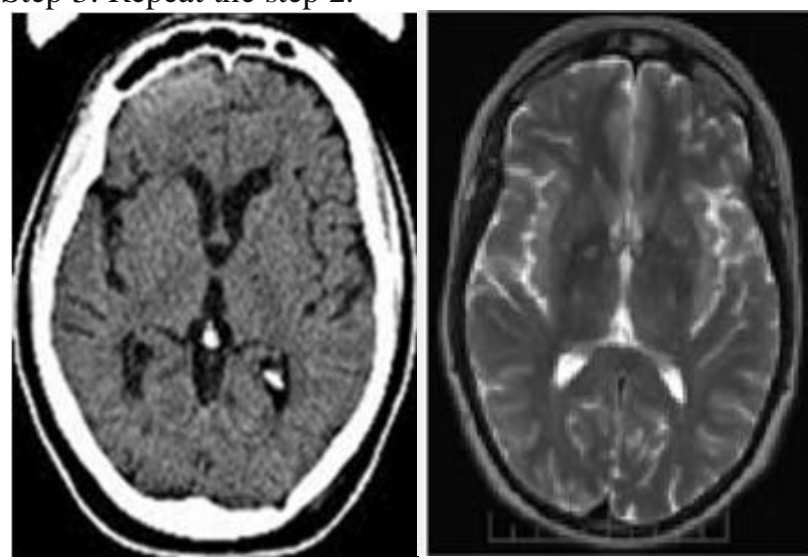

Fig 5 a) Input image b) Contrast stretching output image

\section{D.Clahe}

Contrast limited adaptive histogram equalization has produced good results on medical images. Adaptive Histogram Equalization (AHE) figures the histogram of a focused window at an offered pixel to decide the mapping for that pixel, which gives a nearby complexity upgrade A generalization of AHE, contrast limiting AHE (CLAHE) has more flexibility in choosing the local histogram mapping function.

Step 1: The image has to be divided into several non overlapping regions of almost equal sizes.

Step 2: Compute the histogram of each region is calculated. Step 3: Set a clip limit for clipping histograms.

Step 4: Every histogram is redistributed so that its tallness does not go past as far as possible.
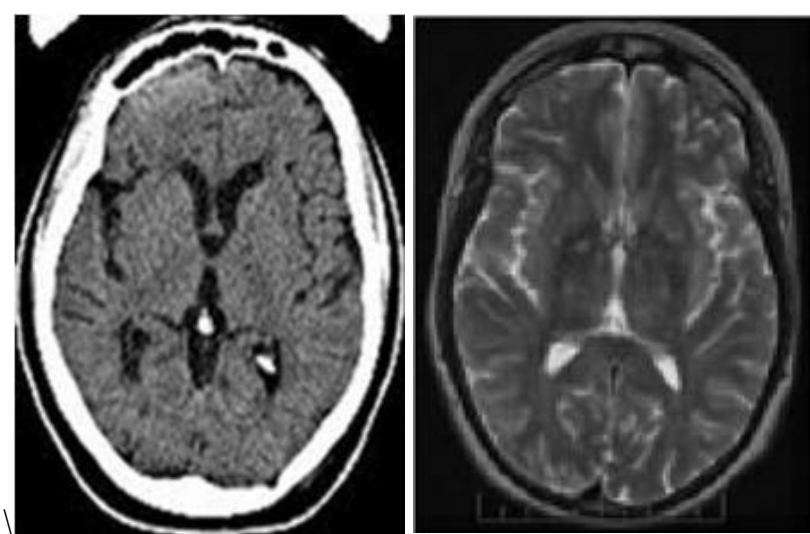

Fig6: a) Input image b) CLAHE output image

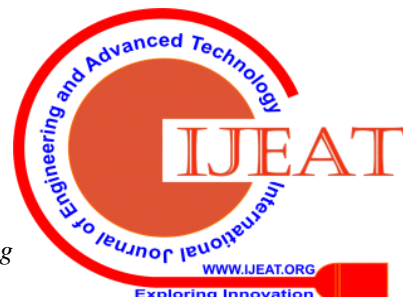




\section{E.Mean filter}

The Mean filter (MNF) is used to eliminate the noise by taking the mean of the neighborhood pixels.MNF is a linear low filter. It works on the concept of windowed filter. In this type of filter centre value window is replaced with the averaged value. The image details are not preserved in this operation, some details are lost. It is a simple to use and easy to implement filter to remove unwanted value of the pixel.
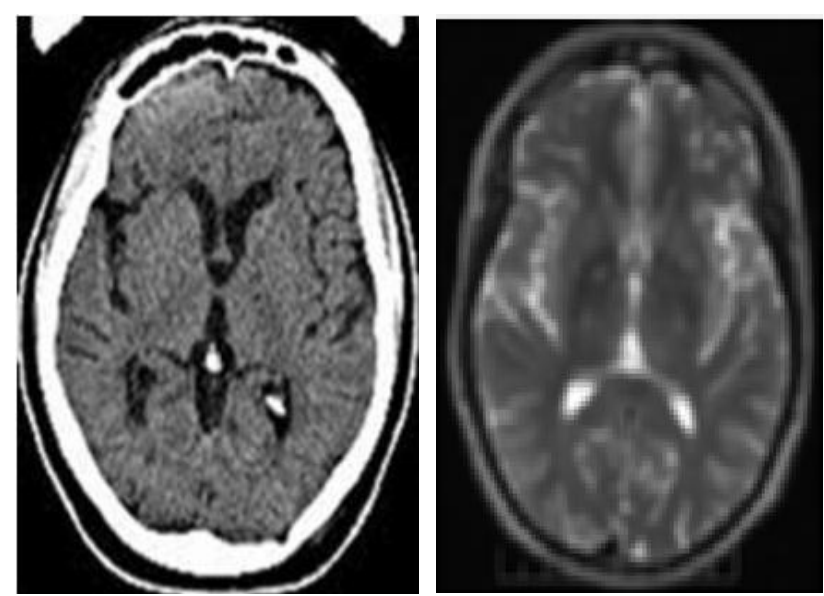

Fig 7 : a) Input image b) Mean output image

\section{F. Median Filtering:}

The Median Filter is a linear digital filtering technique, often used to remove noise from an image or signal. Such noise reduction is a typical pre-processing step to improve the results of later processing. Median filters (MDNF) can remove certain types of noise, especially impulse noise where each pixel has high its own high values.
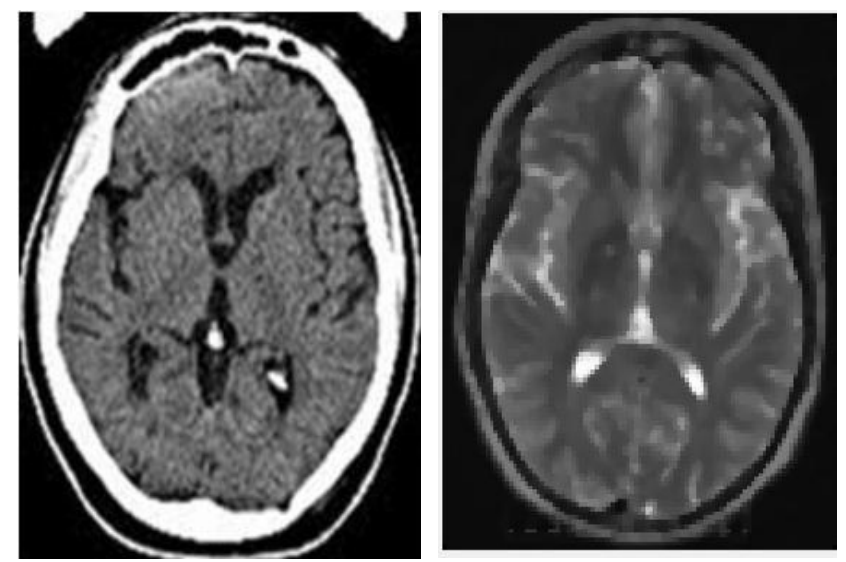

Fig 8 : a) Input image b) Median output image

\section{Review of Spatial Domain Enhancement:}

Various spatial domain techniques are compared in terms of mean, standard deviation and entropy for an brain sample whose values are shown below to analyze that CLAHE method imprints better results. The filtering methods such as mean, median filter are tabulated which indicates filtering with CLAHE methods can be combined in our proposed method to improve the performance of an sample image in terms of removing noises.

\begin{tabular}{|l|l|l|l|}
\hline \multicolumn{1}{|c|}{ Spatial methods } & $\begin{array}{c}\text { Mean } \\
(\mu)\end{array}$ & $\begin{array}{c}\text { Standard } \\
\text { deviation }(\sigma)\end{array}$ & Entropy \\
\hline Unsharp mask & 132 & 55 & 10.2 \\
\hline $\begin{array}{l}\text { Power law } \\
\text { transformation }\end{array}$ & 101.2 & 51 & 7.42 \\
\hline Contrast stretch & 62.2 & 43.4 & 6.68 \\
\hline $\begin{array}{l}\text { Histogram } \\
\text { equalization }\end{array}$ & 128.32 & 73.86 & 5.59 \\
\hline $\begin{array}{l}\text { Filtering } \\
\text { methods(mean, } \\
\text { median) }\end{array}$ & 63.3 & 39.2 & 0.004 \\
\hline CLAHE & 57.2 & 37.15 & 6.6 \\
\hline
\end{tabular}

Tab 1: Comparing the spatial domain enhancement methods

\section{PROPOSED MEHOD}

In the proposed algorithm, we have implemented the methods; mathematical morphology, mean filtering and CLAHE respectively. We obtaining the input image from Brain $\mathrm{O}$ Web datasheet and we are doubling the pixel using matlab.That image is fed into the Histogram Equalization (HE) and then to Adaptive Histogram Equalization(AHE),in order to increase the PSNR value. In CLAHE method, contrast of the image and PSNR value is increased. Sequentially, the noise is reduced by medical filtering method which includes Mean, Median and anisotropic filters. The CLAHE output is providing as input to the median filter. It increases the PSNR and decreases the MSE value in efficient way.

The algorithm of the proposed system is as follows:

STEP1: Input image $512 \mathrm{X} 512$ pixel is obtained

STEP2: Double the image by imdouble

STEP3: Give the image to Histogram Equilization(HE)

STEP4: CLAHE method is computed

STEP5: Contrast of CLAHE is then presented to Medical filtering (i.e. median filtering) method

STEP6: Pertain median filter to the image

STEP7: Increases the PSNR and Decrease the MSE
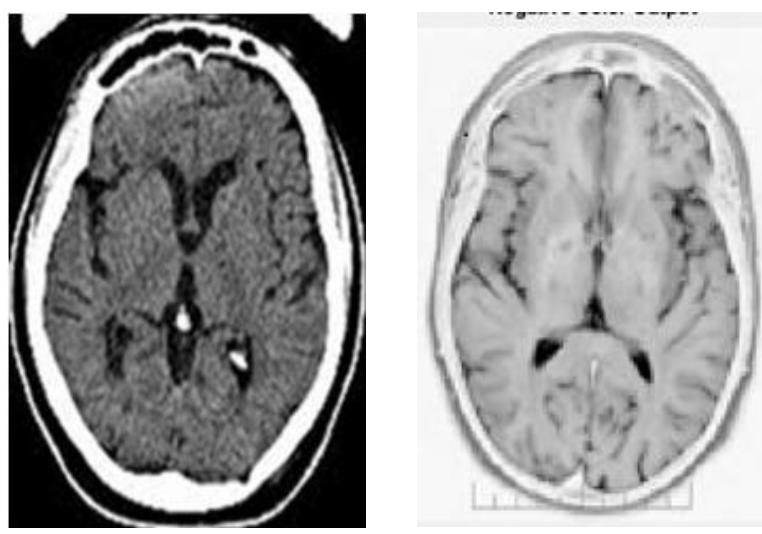

Published By:

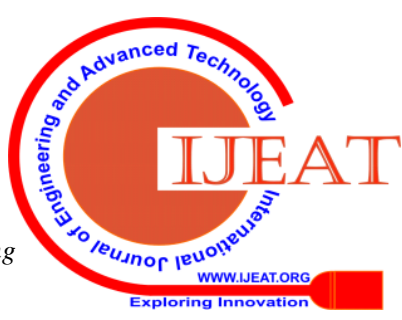




\section{Denoising of MRI Brain Images Using Adaptive Clahe Filtering Method}
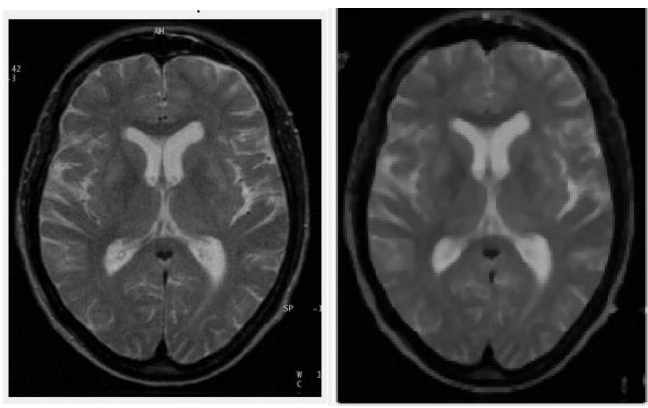

Fig 7 a) pre processed input image b) proposed output image

\section{PERFORMANCE ANALYSIS}

\section{A. Measuring metrices}

In order to compare the effects of image enhancement in the use of different methods, some performance indexes would be used to evaluate the enhancement effect. The commonly used objective measures are mean $(\mu)$,the average of all intensities to determine the average brightness of image, standard deviation $(\sigma)$ the deviation of intensity values about mean value to define average contrast of the image. The two metrics used to compare the quality of image are mean square error (MSE) and PSNR (peak signal to noise ratio).

$$
\text { Standard Deviation: } \sigma=\sqrt{\frac{1}{M N} \sum_{x=1}^{M} \sum_{y=1}^{N}[I(x, y)-\mu]^{2}}
$$

$$
\begin{gathered}
P S N R=10 * \log _{10}\left(\frac{M a x^{2}}{M S E}\right) \\
M S E=\frac{1}{M * N} \sum_{x=0}^{M-1} \sum_{y=0}^{N-1}\left(I_{(x, y)}-R_{(x, y)}\right)^{2} \\
\text { MEAN }: \mu=\frac{1}{M N} \sum_{x=1}^{M} \sum_{y=1}^{N} I(x, y)
\end{gathered}
$$

where $\mathrm{I}(\mathrm{x}, \mathrm{y})$ is the intensity value of pixel $(\mathrm{x}, \mathrm{y})$, and $(\mathrm{M}$,

Tab 1:PSNR values of various techniques with proposed technique.

\begin{tabular}{|c|c|c|c|c|c|c|c|}
\hline $\begin{array}{c}\text { Input } \\
\text { Images }\end{array}$ & LOG & $\begin{array}{c}\text { Contrast } \\
\text { Stretch }\end{array}$ & Negative & CLAHE & HE & MF & $\begin{array}{c}\text { Median } \\
\text { filter }\end{array}$ \\
\hline & & & & & & & \\
1 & 16.4 & 38 & -48.1 & 27.3 & 10.96 & 26.4 & 37.4 \\
\hline 2 & 18.4 & 31.8 & -48.1 & 32.8 & 9.64 & 36.9 & 36.6 \\
\hline 3 & 18.2 & 32.4 & -48.1 & 30.8 & 9.45 & 36.1 & 36.5 \\
\hline 4 & 16.3 & 31.2 & -48.1 & 30.8 & 9.1 & 24.7 & 35.5 \\
\hline 5 & 16.3 & 30.8 & -48 & 31.2 & 9.36 & 2405 & 35.9 \\
\hline
\end{tabular}

Tab 2:MSE values of various techniques with proposed technique.

\begin{tabular}{|c|c|c|c|c|c|c|c|}
\hline $\begin{array}{c}\text { Input } \\
\text { Images }\end{array}$ & LOG & $\begin{array}{c}\text { Contrast } \\
\text { Stretch }\end{array}$ & Negative & CLAHE & HE & MF & $\begin{array}{c}\text { Median } \\
\text { filter }\end{array}$ \\
\hline 1 & 13.7 & 14.3 & 255 & 14.8 & 15.5 & 0.2 & 71.4 \\
\hline 2 & 11.9 & 12.6 & 255.7 & 12.6 & 15.96 & 0.2 & 63.4 \\
\hline 3 & 12.1 & 14 & 255.8 & 13.6 & 15.6 & 0.2 & 59.1 \\
\hline 4 & 12 & 15.2 & 255.8 & 13.6 & 15.5 & 0.2 & 59.1 \\
\hline 5 & 11.8 & 14.9 & 255.6 & 14.2 & 15.36 & 0.2 & 59.3 \\
\hline
\end{tabular}

$\mathrm{N})$ is the dimension of the image.

The higher PSNR shows improved quality image. The lower value of MSE represents lower error. In comparing the different enhancement and denoising techniques the proposed filtering method is effective in improving the contrast as well removing the noise. This is proved from the high PSNR and low MSE values obtained from the various brain images used. The results of differensamples are shown in the below table 1 and table 2 .

Table 1 shows the PSNR values of different samples where table 2 compares the MSE values of same samples.

\section{RESULTS AND DISCUSSION}

The results of brain dataset obtained are analyzed by its mean,PSNR, and MSE values the input and its corresponding output samples are shown below where fig a is the input sample fig $b$ is contrast sterch fig c power law fig $\mathrm{d}$ is $\mathrm{HE}$ fig e is negative fig $\mathrm{f}$ is mean fig $\mathrm{g}$ is median fig $\mathrm{h}$ is proposed ACLAHE Filter
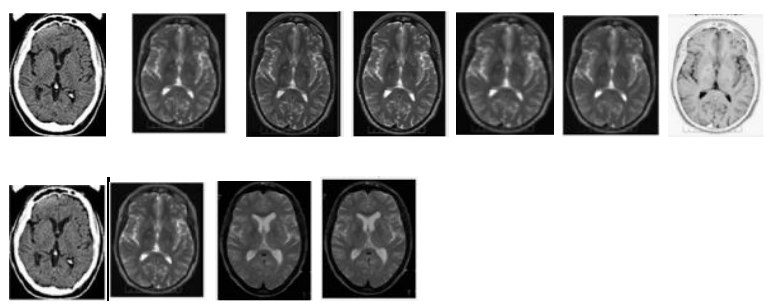

fig a) input sample, fig b)contrast sterch, fig c) power law fig d) $\mathrm{HE}$,fig e) negative, figf) mean, fig $g$ ) median,

fig $h$ ) proposed ACLAHE Filter

\section{CONCLUSION}

This paper is concentrated for analying the denoising using filtering technique.It implements the median and Contrast limited adaptive method to eliminate the noise and modifying or restore an image without resolution loss.

\section{REFERENCES}

1. Rafael C. Gonzalez, and Richard E. Woods, "Digital Image Processing", 2nd edition, Prentice Hall, 2002.

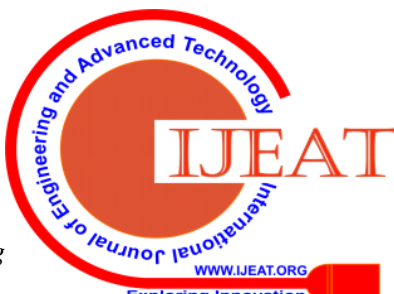


2. Arici,"A Histogram modification framework and its application for image contrast enhancement",IEEE transcation on image processing ,2009

3. Nymlkhagva Sengee, and Heung Kook Choi, "Brightness preserving weight clustering histogram equalization”, IEEE Trans. Consumer Electronics, vol. 54, no. 3, pp. 1329 - 1337, August 2008

4. K.Jain ,'Fundamentals of digital image processing", Englewood's cliffs: Prentice -hall 1997.

5. P.G.Akila,Dr.K.Batri,"survey on different preprocessing methods of enhancement and segmentation in medical image analysis"Journal of advanced research in dynamical and control systems,vol 10,2018.

6. Beilei Xu, Yiqi Zhuang Hualian Tang,Li Zhang, "Object-Based Multilevel Contrast Stretching Method for Image Enhancement" IEEE Transactions on Consumer Electronics, Vol. 56, No. 3, Aug 2010

7. Andrea Polesel, Giovanni Ramponi, and V. John Mathews, "Image Enhancement via Adaptive Unsharp Masking" IEEE transactions on image processing, vol. 9, no. 3, march 2000

8. Showkat Hassan Malik ,Mewar S.M.K. Quadri "Contras Enhancement and Smoothing of CT Images for Diagnosis" International Conference on Computing for Sustainable Global Development ISSN-3805-4416-8/15 2015 IEEE

9. Sudhir Kumar Suman, B.Ganesan and G. Yamuna, "Hybrid Contrast Enhancement Approach for Medical Images",International Journal of Computer Applications (IJCA), pp. 9-12.

10. Elena S. Yelmanova , M. Romanyshyn"Medical Image Contrast Enhancement Based on Histogram "2017 IEEE International Conference on Electronics and Nanotechnology ISSN--53861701 vol-4.

11. Faisel G.Mohammed, Sega G. Mohammed, "Contrast \& Brightness Enhancement for Low Medical X-Ray Images", International Journal of Scientific \& Engineering Research,Volume 4, Issue 5, May-2013.

12. G.N.Sarage,SagarJambhorkar, "Enhancement of Chest X-Ray images Using Filtering Techniques",Volume 2,Issue 5 May 2012 2277 128X.

13. Ying Shen, WeihuaZhu,"Medical Image Processing using A Machine Vision based Approach", International Journal of Signal Processing, Image Processing and Pattern Recognition Vol. 6, No. 3 June, 2013.

14. Mandip Kaur and Richa Sharma,"Restoration of Medical Images Using Denoising", International Journal for Science and Emerging Technologies with Latest Trends "5(1): 35-38 (2013) ISSN No. 2277-8136

15. Md. Moniruzzaman , Md. Shafuzzaman and Md. Foisal Hossain, "Detailed Regions Based Medical Image Contrast Enhancement " International Conference on Advances in Electrical Engineering ISSN -4799-2465-3/13 IEEE

\section{AUTHOR PROFILE}

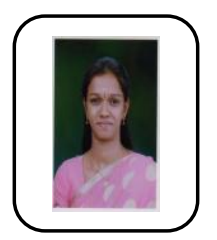

Mrs.P.G.Akila received the M.E degree in applied electronics from PSNA College of Engineering and Technology, Anna University, Chennai, in 2009. She is currently pursuing the Ph.D. degree in Medical image analysis at Anna University, Chennai, under the supervision of Prof. Dr.K.Batri. Her research interests include Digital Image Processing, Pattern Recognition and Artificial Neural Networks. She is currently an Assistant Professor in PSNA College of Engineering and Technology, Anna University Chennai .She has published more than 20 papers in top tier academic journals and conferences. She has completed many online courses in machine learning and image processing. She is an active member of various professional societies like ISTE, IEEE, and IAENG etc. 